\title{
LOCAL COMPACTNESS IN FREE TOPOLOGICAL GROUPS
}

\author{
Peter Nickolas and Mikhail TKachenko
}

\begin{abstract}
We show that the subspace $A_{n}(X)$ of the free Abelian topological group $A(X)$ on a Tychonoff space $X$ is locally compact for each $n \in \omega$ if and only if $A_{2}(X)$ is locally compact if and only if $F_{2}(X)$ is locally compact if and only if $X$ is the topological sum of a compact space and a discrete space. It is also proved that the subspace $F_{n}(X)$ of the free topological group $F(X)$ is locally compact for each $n \in \omega$ if and only if $F_{4}(X)$ is locally compact if and only if $F_{n}(X)$ has pointwise countable type for each $n \in \omega$ if and only if $F_{4}(X)$ has pointwise countable type if and only if $X$ is either compact or discrete, thus refining a result by Pestov and Yamada. We further show that $A_{n}(X)$ has pointwise countable type for each $n \in \omega$ if and only if $A_{2}(X)$ has pointwise countable type if and only if $F_{2}(X)$ has pointwise countable type if and only if there exists a compact set $C$ of countable character in $X$ such that the complement $X \backslash C$ is discrete. Finally, we show that $F_{2}(X)$ is locally compact if and only if $F_{3}(X)$ is locally compact, and that $F_{2}(X)$ has pointwise countable type if and only if $F_{3}(X)$ has pointwise countable type.
\end{abstract}

\section{INTRODUCTION}

Understanding the topological properties of free and free Abelian topological groups is one of the main objectives in the study of topological groups in general. It is well known that the groups $F(X)$ and $A(X)$, the free topological group over a Tychonoff space $X$ and its Abelian analogue, respectively, are metrisable if and only if the space $X$ is discrete [6, Section 6, Statement C]. Similarly, the groups $F(X)$ and $A(X)$ are locally compact if and only if $X$ is discrete. The latter follows from the more general result of Dudley [4] that the only locally compact Hausdorff topological group topology on an abstract free (or free Abelian) group is the discrete topology. The result also follows, at least in the non-Abelian case, from the observation of Arhangel'skii [2] that if $F(X)$ is a Baire space, then $X$ is discrete. We add almost metrisability to the list of properties in free topological groups implying discreteness: if one of the groups $F(X)$ or $A(X)$ is almost metrisable, then $X$ is discrete (see Proposition 4.2).

Received 12th February, 2003

The second author wishes to thank the first author, and his department, for hospitality extended during the course of this work, and acknowledges the financial support of a Quality Fund grant from the Institute for Mathematical Modelling and Computational Systems (IMMaCS) at the University of Wollongong.

Copyright Clearance Centre, Inc. Serial-fee code: 0004-9727/03 \$A2.00+0.00. 
For every non-negative integer $n$, let $F_{n}(X)$ be the subset of $F(X)$ which consists of all elements having reduced length less than or equal to $n$ with respect to the basis $X$, and define the subset $A_{n}(X)$ of $A(X)$ in a similar way. Yamada proved in [22, Theorem 4.9] that $F_{n}(X)$ is metrisable for each integer $n \in \omega$ if and only if $F_{4}(X)$ is metrisable if and only if $X$ is compact metrisable or discrete. The Abelian case is different: $A_{n}(X)$ is metrisable for each integer $n$ if and only if $A_{2}(X)$ is metrisable if and only if $X$ is metrisable and the set $X^{\prime}$ of non-isolated points of $X$ is compact. We conclude in particular that the metrisability of all finite levels $F_{n}(X)$ of the group $F(X)$ does not imply the metrisability of $F(X)$, and that neither does the metrisability of the levels $A_{n}(X)$ imply that of $A(X)$. (One can, however, see this easily by a direct argument, since results known to Graev [6] show that if $X$ is an infinite compact metric space, then the finite levels of $F(X)$ and $A(X)$ are compact and metrisable, while the groups themselves are not metrisable.)

The local compactness of the spaces $F_{n}(X)$ was considered by Yamada under the additional assumption that $X$ is metrisable. He showed in [21, Proposition 3.3] that, under this assumption, the levels $F_{n}(X)$ are locally compact if and only if $X$ is either compact or discrete. Afterwards, Pestov and Yamada proved in [15] that, for an arbitrary Tychonoff space $X$, if each $F_{n}(X)$ is locally compact, then $X$ is discrete or pseudocompact. In fact, pseudocompactness in this assertion can be strengthened to countable compactness [15, Coroollary 3.6]. Here we show that the spaces $F_{n}(X)$ are locally compact for all $n \in \omega$ if and only if $F_{4}(X)$ is locally compact if and only if $X$ is either discrete or compact, thus finishing the work started in $[21,15]$. Exactly the same conclusion remains valid if one weakens local compactness to pointwise countable type (see Theorem 2.9). It turns out that $n=4$ is the minimal integer with this property, in both cases: by Theorem 2.13 and Proposition $2.14, F_{2}(X)$ is locally compact if and only if $F_{3}(X)$ is locally compact if and only if $X$ is the topological sum of a compact space and a discrete space, and $F_{2}(X)$ has pointwise countable type if and only if $F_{3}(X)$ has pointwise countable type if and only if there exists a compact set $C$ of countable character in $X$ such that the points of the complement $X \backslash C$ are isolated in $X$ (see Theorem 3.3 and Proposition 3.7). Further, $F_{2}(X)$ is locally compact if and only if $A_{2}(X)$ is locally compact if and only if $A_{n}(X)$ is locally compact for all $n \in \omega$, with a similar equivalence holding in the case of pointwise countable type (Theorems 2.13 and 3.5 ). Finally, it turns out that $F_{2}(X)$ has pointwise countable type if and only if $F_{2}(X)$ is Cech-complete, and the same equivalence holds in the Abelian case (Theorem 3.8).

This article is organised as follows. Section 1.1 introduces notation and terminology used throughout the paper. In Section 1.2 we present in summary form a few basic results about free (Abelian) topological groups which have several applications in the main body of the article. The local compactness and pointwise countable type of the finite levels of the groups $F(X)$ and $A(X)$ are studied in Section 2. We show in Lemma 2.1 that if 
$F_{2}(X)$ has pointwise countable type, then the set $X^{\prime}$ of all non-isolated points of $X$ is bounded in $X$. After several steps, in Corollary 2.7, we strengthen this result and deduce that the set $X^{\prime}$ is compact. Almost all subsequent results essentially lean on this fact. In Section 3, we characterise the spaces $X$ such that $F_{2}(X)$ and $A_{2}(X)$ have pointwise countable type or are Čech-complete.

1.1. NOTATION AND TERMINOLOGY, In what follows, all spaces are assumed to be Tychonoff. For a space $X$, the derived set $X^{\prime}$ is the set of all non-isolated points of $X$. Obviously, $X^{\prime}$ is closed in $X$. A subset $Y$ of a space $X$ is called $P$-embedded in $X$ if every continuous bounded pseudometric on $Y$ extends to a continuous pseudometric on $X$. We also say that $Y$ is bounded in $X$ if $f(Y)$ is a bounded subset of the real line $\mathbb{R}$, for every continuous function $f: X \rightarrow \mathbb{R}$. A subset $Y$ of $X$ is $\omega$-bounded in $X$ if, for every continuous pseudometric $d$ on $X$ and every $\varepsilon>0$, the set $Y$ can be covered by countably many open balls of radius $\varepsilon$ with respect to $d$. It is clear that every $\sigma$-compact subset of $X$ is $\omega$-bounded in $X$.

We say that a subset $Y$ of a space $X$ is $G_{\delta}$-dense in $X$ if every non-empty $G_{\delta}$-set in $X$ intersects $Y$. It is well-known that every space $X$ is $G_{\delta}$-dense in its Dieudonné completion $\mu X$ (see [5, Chapter 8]).

Suppose that a space $X$ is the union of an increasing sequence $\left\{K_{n}: n \in \omega\right\}$ of compact subsets $K_{n}$. If a subset $Y$ of $X$ is closed in $X$ if and only if all intersections $Y \cap K_{n}$ are closed in $X$, then we say that $X=\bigcup_{n \in \omega} K_{n}$ is a $k_{\omega}$-decomposition of $X$. If a space $X$ admits a $k_{\omega}$-decomposition, we call it a $k_{\omega}$-space. All $k_{\omega}$-spaces are obviously $\sigma$-compact, but not vice versa (the space of rationals is a counterexample).

If $Y$ is a subspace of $X$, then $\chi(Y, X)$ denotes the character of $Y$ in $X$, that is, the minimum cardinal of a base at $Y$ in $X$.

The free topological group over a space $X$ is denoted by $F(X)$, and $A(X)$ stands for the free Abelian topological group over $X$. Given a subset $Y$ of $X$, we use $F(Y, X)$ to denote the subgroup of $F(X)$ generated by $Y$, while $A(Y, X)$ stands for the corresponding subgroup of $A(X)$. For every integer $n \in \omega, F_{n}(X)$ is the subset of $F(X)$ consisting of the words having reduced length $\leqslant n$ with respect to $X$, and $A_{n}(X)$ is the corresponding subset of $A(X)$. It is well known that the sets $F_{n}(X)$ are closed in $F(X)$ and that the sets $A_{n}(X)$ are closed in $A(X)$ for all $n \in \omega$ (see [6] or [3]). We shall use additive notation for the sum operation in $A(X)$.

Let $e$ be the identity of the free topological group $F(X)$. The subspace $F_{1}(X)$ of $F(X)$ is naturally homeomorphic to the topological sum $\tilde{X}=X \oplus\{e\} \oplus X^{-1}$. Clearly, the multiplication mapping $j_{n}: \widetilde{X}^{n} \rightarrow F_{n}(X)$ defined by $j_{n}\left(x_{1}, \ldots, x_{n}\right)=x_{1} \cdots x_{n}$ is continuous for each $n \geqslant 1$, and $j_{n}\left(\tilde{X}^{n}\right)=F_{n}(X)$. The inverse image of an element $g \in F_{n}(X)$ under $j_{n}$ is denoted by $j_{n}^{\leftarrow}(g)$. Observe that $j_{n}^{\leftarrow}(g)$ is a singleton for each $g \in F_{n}(X) \backslash F_{n-1}(X)$. 
The subgroup of a group $G$ generated by a set $A \subseteq G$ is $\langle A\rangle$, and $\langle g\rangle$ is the cyclic subgroup of $G$ generated by an element $g \in G$. The fact that $H$ is a subgroup of $G$ abbreviates to $H \leqslant G$. If $A$ is a subset of a group $G$, then we use $A^{n}$ to denote the set $\underbrace{A \cdots A}_{n \text { times }}$. In the Abelian case, $A^{n}$ is replaced by $n A$.

1.2. Preliminaries. For a space $X$, denote by $\mathcal{P}(X)$ the family of all continuous pseudometrics on $X$. Given a pseudometric $d \in \mathcal{P}(X)$, we use $\widehat{d}$ and $\hat{d}_{A}$ to denote Graev's extension of $d$ to a maximal invariant pseudometric on $F(X)$ and $A(X)$, respectively [6]. The pseudometric $\hat{d}$ is continuous on $F(X)$, so the set

$$
O(d)=\{g \in F(X): \widehat{d}(e, g)<1\}
$$

is open in $F(X)$. In particular, the set

$$
O_{2}(d)=O(d) \cap F_{2}(X)=\left\{x^{\delta} y^{-\delta}: x, y \in X, d(x, y)<1, \delta= \pm 1\right\}
$$

is an open neighbourhood of $e$ in $F_{2}(X)$. In general, the family $\{O(d): d \in \mathcal{P}(X)\}$ is not a base at the identity of the group $F(X)$, even if $X$ is compact. The next result, proved by Pestov [14], shows that the situation changes for the subspace $F_{2}(X)$ of $F(X)$.

THEOREM 1.1. For an arbitrary space $X$, the family $\left\{O_{2}(d): d \in \mathcal{P}(X)\right\}$ is a base for $F_{2}(X)$ at the point $e$.

As in the non-Abelian case, the pseudometrics $\hat{d}_{A}$ are continuous on $A(X)$, so the set

$$
O^{a}(d)=\left\{g \in A(X): \widehat{d}_{A}(0, g)<1\right\}
$$

is open in $A(X)$ for each $d \in \mathcal{P}(X)$, where 0 is the neutral element of $A(X)$. The following theorem shows one of the principal differences between the groups $F(X)$ and $A(X)$ (see $[11,14,18])$ :

Theorem 1.2. For every space $X$, the family $\left\{O^{a}(d): d \in \mathcal{P}(X)\right\}$ forms a base at the neutral element 0 of $A(X)$.

An elementary calculation shows that, for each $d \in \mathcal{P}(X)$,

$$
O_{2}^{a}(d)=O^{a}(d) \cap A_{2}(X)=\{x-y: x, y \in X, d(x, y)<1\}
$$

Therefore, we have the following:

Corollary 1.3. For any space $X$, the family $\left\{O_{2}^{a}(d): d \in \mathcal{P}(X)\right\}$ is a base at the point 0 of $A_{2}(X)$.

A useful description of a neighbourhood base at the neutral element of $A(X)$ in terms of uniform entourages of the diagonal in $X^{2}$ is as follows (see [22]). Let $\mathcal{U}_{X}$ be the finest uniformity on $X$ compatible with the topology of $X$ (such a uniformity is called 
the universal uniformity of $X\left[5\right.$, Chapter 8]). For every element $U \in \mathcal{U}_{X}$, denote by $\widetilde{U}$ the set $\{x-y:(x, y) \in U\} \subseteq A_{2}(X)$. Since every uniformity on $X$ compatible with the topology of $X$ is generated by a family of continuous pseudometrics on $X$, it follows from Corollary 1.3 that $\widetilde{U}$ is open in $A_{2}(X)$ for each $U \in \mathcal{U}_{X}$ and the family $\left\{\widetilde{U}: U \in \mathcal{U}_{X}\right\}$ is a base for $A_{2}(X)$ at the element 0 . Let $s: \omega \rightarrow \mathcal{U}_{X}$ be an arbitrary function, so that $s(n) \in \mathcal{U}_{X}$ for each $n \in \omega$. We put

$$
O(s)=\left\{\left(x_{1}-y_{1}\right)+\cdots+\left(x_{n}-y_{n}\right):\left(x_{i}, y_{i}\right) \in s(i) \text { for each } i=1, \ldots, n, n \geqslant 1\right\} .
$$

THEOREM 1.4. The family $\left\{O(s): s \in\left(\mathcal{U}_{X}\right)^{\omega}\right\}$ is a local base at the neutral element of the group $A(X)$.

The next result from $[1,8,7]$ provides important information about the topological structure of the subspaces $F_{n}(X) \subseteq F(X)$ and $A_{n}(X) \subseteq A(X)$ of elements of length precisely $n$.

Proposition 1.5. Let $g=x_{1}^{\varepsilon_{1}} \cdots x_{n}^{\varepsilon_{n}}$ be an irreducible element of $F_{n}(X)$, where $x_{1}, \ldots, x_{n} \in X$ and $\varepsilon_{1}, \ldots, \varepsilon_{n}= \pm 1$. Then the sets of the form $U_{1}^{\varepsilon_{1}} \cdots U_{n}^{\varepsilon_{n}}$ constitute a base for $F_{n}(X)$ at $g$, where $U_{i}$ is an open neighbourhood of $x_{i}$ for $i=1, \ldots, n$ and $U_{i} \cap U_{j}=\emptyset$ if $x_{i} \neq x_{j}$. Hence the mapping $j_{n}: \tilde{X}^{n} \rightarrow F_{n}(X)$ is a local homeomorphism at every point $a \in \widetilde{X}^{n}$ whose image $j_{n}(a)$ has reduced length exactly $n$. Similarly, the sets of the form $\varepsilon_{1} U_{1}+\cdots+\varepsilon_{n} U_{n}$ constitute a base at the irreducible element $g=\varepsilon_{1} x_{1}+\cdots+\varepsilon_{n} x_{n}$ in $A_{n}(X)$.

One further useful fact about free Abelian topological groups, which follows from general categorical considerations, is given below (see for example [10]).

Proposition 1.6. Let $X=X_{1} \oplus X_{2}$ be the topological sum of spaces $X_{1}$ and $X_{2}$. Then $A(X) \cong A\left(X_{1}\right) \times A\left(X_{2}\right)$.

The subgroup $F(Y, X)$ of $F(X)$ generated by a closed subset $Y$ of $X$ is always closed in $F(X)$ [6, Section 4, Statement D]. It is clear that the natural isomorphism $i_{Y, X}$ of $F(Y)$ onto $F(Y, X)$ is continuous, but it is not necessarily a homeomorphism. In other words, the topology of the group $F(Y, X)$ can be coarser than that of $F(Y)$. The next general result was proved by Uspenskij in [20].

Theorem 1.7. If a subspace $Y$ of a space $X$ is $P$-embedded in $X$ and $\omega$ bounded, then the natural isomorphism $i_{Y, X}$ of $F(Y)$ onto $F(Y, X)$ is a homeomorphism. Conversely, if $i_{Y, X}$ is a homeomorphism, then $Y$ is $P$-embedded in $X$.

In fact, the phrase " $\omega$-bounded" can be omitted in Theorem 1.7 as was shown by Sipacheva [17]. Therefore, $i_{Y, X}$ is a homeomorphism if and only if $Y$ is $P$-embedded in $X$. One very special case when $i_{Y, X}$ is a homeomorphism deserves mention here. It has a very simple, direct proof, so we reproduce it here (see also [19]).

PRoposition 1.8. If $Y$ is a retract of a space $X$, then $i_{Y, X}$ is a homeomorphism. 
Proof: Let $r: X \rightarrow Y$ be a continuous retraction of $X$ onto $Y \subseteq X$. Extend $r$ to a continuous homomorphism $\widehat{r}: F(X) \rightarrow F(Y)$. Then the restriction of $\widehat{r}$ to the subgroup $F(Y, X)$ of $F(X)$ is a continuous isomorphism of $F(Y, X)$ onto $F(Y)$, and the inverse of this restriction is exactly the isomorphism $i_{Y, X}$, which is always continuous. Hence $i_{Y, X}$ is a homeomorphism.

\section{Main Results}

To characterise the local compactness and pointwise countable type of the subspaces $F_{n}(X)$ of $F(X)$, we need a series of lemmas.

LEMMA 2.1. If $F_{2}(X)$ is of pointwise countable type, then the derived set $X^{\prime}$ is bounded in $X$.

Proof: From the hypothesis, there exist in $F_{2}(X)$ a compact set $K$ containing the identity $e$ and a decreasing family of open sets $\left\{U_{n}: n \in \omega\right\}$ which form a base for $F_{2}(X)$ at $K$. Let us assume that $X^{\prime}$ is not bounded in $X$. Then there exists in $X$ a discrete family of open sets $\left\{V_{n}: n \in \omega\right\}$ each of which intersects $X^{\prime}$. Choose a point $x_{n} \in V_{n} \cap X^{\prime}$ for all $n \in \omega$. It is clear that, from the fact that $x_{n}$ is non-isolated, we can find $y_{n} \in V_{n}$ such that $x_{n} \neq y_{n}$ and such that $x_{n}^{-1} y_{n} \in U_{n}$ for all $n$. Note that we also have $x_{m}^{-1} y_{m} \neq x_{n}^{-1} y_{n}$ for distinct $m, n \in \omega$. Set $P=\left\{x_{n}^{-1} y_{n}: n \in \omega\right\}$. We claim that $P$ is closed and discrete in $F_{2}(X)$.

Certainly, $P$ has no accumulation points in $X \cup X^{-1}$, because the set $X \cup X^{-1}$ is open in $F_{2}(X)$ and all elements of $P$ have length 2 . Since the family $\left\{V_{n}: n \in \omega\right\}$ is discrete and $x_{n}$ and $y_{n}$ are distinct points in $V_{n}$, we can find a continuous pseudometric $d$ on $X$ such that $d\left(x_{n}, y_{n}\right) \geqslant 1$ for each $n \in \omega$. Then $O_{2}(d)$ is an open neighbourhood of $e$ in $F_{2}(X)$ disjoint from $P$. Finally, the natural mapping $j_{2}: \widetilde{X}^{2} \rightarrow F_{2}(X)$ is a local homeomorphism of $\widetilde{X}^{2}$ to $F_{2}(X)$ at every point of $\widetilde{X}^{2} \backslash j_{2}^{\leftarrow}(e)$, and the set $L=\left\{\left(x_{n}^{-1}, y_{n}\right): n \in \omega\right\}$ is closed in $\tilde{X}^{2}$ and discrete. Hence $P=j_{2}(L)$ has no accumulation points in $F_{2}(X) \backslash F_{1}(X)$. It follows that $P$ is closed in $F_{2}(X)$ and discrete, as claimed.

Now $K$ cannot contain an infinite subset of $P$, since no such subset has a cluster point. Therefore, there is an infinite subset $P_{0}$ of $P$ such that $P_{0} \cap K=\emptyset$. But then $F_{2}(X) \backslash P_{0}$ is an open set containing $K$ but not containing $U_{n}$ for any $n \in \omega$, a contradiction. We conclude that the set $X^{\prime}$ is bounded in $X$, as required.

The next result is well known in the topological folklore, but it is difficult to find its proof in the literature. This is why the corresponding proof appears here.

LEMMA 2.2. Let $Y$ be a dense subspace of a space $X$. Then $\chi(K, Y)=\chi(K, X)$ for every compact set $K \subseteq Y$.

Proof: The inequality $\chi(K, Y) \leqslant \chi(K, X)$ is obvious, so we only prove the inverse one. Let $\gamma$ be a base for $Y$ at $K$ satisfying $|\gamma|=\chi(K, Y)$. For every $O \in \gamma$, choose 
an open set $U_{O}$ in $X$ such that $U_{O} \cap Y=O$. Then the family $\mu=\left\{U_{O}: O \in \gamma\right\}$ is a base for $X$ at $K$. Indeed, take an arbitrary open neighbourhood $V$ of $K$ in $X$. Then, by the compactness of $K$, there exists another open neighbourhood $W$ of $K$ in $X$ such that $c l_{X}(W) \subseteq V$. Choose $O \in \gamma$ such that $O \subseteq W \cap Y$. Since $Y$ is dense in $X$ and $U_{O} \cap Y=O$, we have $c l_{X}(O)=c l_{X}\left(U_{O}\right)$. Therefore,

$$
K \subseteq U_{O} \subseteq c l_{X}\left(U_{O}\right)=c l_{X}(O) \subseteq c l_{X}(W) \subseteq V .
$$

So, $\mu$ is a base for $X$ at $K$ and $\chi(K, X) \leqslant|\mu| \leqslant|\gamma|=\chi(K, Y)$. This finishes the proof.

The next fact is almost evident.

LEMMA 2.3. Suppose that the set $X^{\prime}$ of non-isolated points in $X$ is compact. Then $X$ is paracompact.

Proof: Let $\gamma$ be an open cover of $X$. By the compactness of $X^{\prime}$, there exists a finite subfamily $\mu$ of $\gamma$ which covers $X^{\prime}$. Then $\nu=\mu \cup\{\{x\}: x \in \cup \mu\}$ is an open locally finite cover of $X$ which refines $\gamma$. Hence $X$ is paracompact.

REMARK 2.4. We do not need the fact here, but we note that a straightforward inductive argument can be given to show that, in Lemma 2.3, the space $X^{n}$ is paracompact for each $n \geqslant 1$.

Following [5], we denote by $\mu X$ the Dieudonné completion of a space $X$. We recall that $X^{\prime}$ is the set of non-isolated points in $X$.

LEMMA 2.5. Suppose that $X^{\prime} \subseteq Y \subseteq X$ and that $Y$ is bounded in $X$. Then the set $K=c l_{\mu X} Y$ is compact and $\mu X=X \cup K$. In addition, $Y$ is $G_{\delta}$-dense in $K$.

Proof: The set $K$ is compact since the space $\mu X$ is Dieudonné complete and $Y$ is bounded in both $X$ and $\mu X$. Put $Z=X \cup K$. Then $X \subseteq Z \subseteq \mu X$, and Lemma 2.3 implies that the space $Z$ is paracompact and, hence, Dieudonné complete. Since a space $T$ with $X \subseteq T \subseteq \mu X$ is Dieudonné complete if and only if $T=\mu X$ (see [5, Chapter 8]), we conclude that $Z=\mu X$. The equality $\mu X=X \cup K$ is now immediate.

To prove the second claim of the lemma, suppose that there exists a non-empty $G_{\delta^{-}}$ set $P$ in $K$ disjoint from $Y$. Let $P=\bigcap_{n \in \omega} U_{n}$, where each $U_{n}$ is open in $K$. Pick a point $x_{0} \in P$. Clearly, $x_{0}$ is non-isolated in $\mu X$. Then there exists a sequence $\left\{V_{n}: n \in \omega\right\}$ of open sets in $\mu X$ such that $x_{0} \in V_{n}, V_{n} \cap K \subseteq U_{n}$ and $c_{\mu X}\left(V_{n+1}\right) \subseteq V_{n}$ for each $n \in \omega$. Since $x_{0} \in P \subseteq K$, each $V_{n}$ intersects $Y$. For every $n \in \omega$, choose a point $y_{n} \in V_{n} \cap Y$ in such a way that $y_{n} \neq y_{m}$ if $n \neq m$. From our choice of the sets $V_{n}$ it follows that all accumulation points of the set $S=\left\{y_{n}: n \in \omega\right\}$ lie in $P \subseteq K \backslash Y$, so $S$ is closed and discrete in both $Y$ and $X$ (note that $Y$ is closed in $X$ ). Clearly, there exists a disjoint family $\gamma=\left\{W_{n}: n \in \omega\right\}$ of open sets in $X$ such that $y_{n} \in W_{n} \subseteq V_{n}$ for each $n \in \omega$. We claim that the family $\gamma$ is locally finite in $X$. 
Indeed, the disjoint family $\gamma$ cannot have accumulation points in $X \backslash Y$ since all points of the latter set are isolated in $X$. Let $y \in Y$ be arbitrary. Then $y \notin U_{n}$ for some $n \in \omega$, so $O=X \backslash c l_{\mu X}\left(V_{n+1}\right)$ is an open neighbourhood of $y$ in $X$. Since $W_{k+1} \subseteq V_{k+1} \subseteq V_{k}$ for each $k \in \omega$, the set $O$ does not intersect $W_{k}$ if $k>n$. This implies that the family $\gamma$ has no accumulation points in $Y$ and, hence, it is locally finite in $X$, thus proving the claim.

Finally, each element of $\gamma$ intersects the set $Y$, which contradicts the boundedness of $Y$ in $X$. Therefore, $Y$ is $G_{\delta}$-dense in $K$.

LEMMA 2.6. If $F_{2}(X)$ has pointwise countable type, then every closed bounded subset of $X$ is compact.

Proof: Suppose that $F_{2}(X)$ is of pointwise countable type and take a compact set $K \subseteq F_{2}(X)$ which contains the identity $e$ of $F(X)$ and satisfies $\chi\left(K, F_{2}(X)\right) \leqslant \omega$. By the Nummela-Pestov theorem (see [12, 14]), $F(X)$ can be identified with the subgroup $F(X, \mu X)$ of $F(\mu X)$. Then $F_{2}(X)$ is identified with the subspace $F_{2}(\mu X)$

$\cap F(X)$ of $F_{2}(\mu X)$. Observe that $F_{2}(X)$ is dense in $F_{2}(\mu X)$ since $X$ is dense in $\mu X$. Hence Lemma 2.2 implies that $\chi\left(K, F_{2}(\mu X)\right) \leqslant \omega$.

Let $\left\{U_{n}: n \in \omega\right\}$ be a countable base for $F_{2}(\mu X)$ at $K$. By Theorem 1.1, for every $n \in \omega$ there exists a continuous pseudometric $d_{n}$ on $\mu X$ such that $O_{2}\left(d_{n}\right) \subseteq U_{n}$. We can assume without loss of generality that each $d_{n}$ is bounded by 1 . Then $d=\sum_{n=0}^{\infty} 2^{-n} d_{n}$ is a continuous pseudometric on $\mu X$ and one easily verifies that the set

$$
P=\left\{x^{-1} y: x, y \in \mu X, d(x, y)=0\right\}
$$

satisfies $P \subseteq \bigcap_{n \in \omega} O_{2}\left(d_{n}\right) \subseteq \bigcap_{n \in \omega} U_{n}=K$. In particular, $P \subseteq F_{2}(X)$

Let $B$ be a closed bounded subset of $X$. Since the derived set $X^{\prime}$ is bounded in $X$ by Lemma 2.1, the union $Y=X^{\prime} \cup B$ is also bounded in $X$. Hence the set $C=c_{\mu X}(Y)$ is compact. As $B$ is closed in $Y$, it suffices to show that $Y=C$. Suppose not, and choose a point $x_{0} \in C \backslash Y$. Obviously, $F=\left\{y \in \mu X: d\left(x_{0}, y\right)=0\right\}$ is a $G_{\delta}$-set in $\mu X$ and $x_{0} \in F$. Since $Y$ is $G_{\delta}$-dense in $C$ by Lemma 2.5 , we can choose a point $y_{0} \in F \cap Y$. Then $x_{0}^{-1} y_{0} \in P \backslash F_{2}(X)$, which contradicts the inclusion $P \subseteq F_{2}(X)$ established above. Hence $Y$ is compact.

Combining Lemmas 2.1 and 2.6, we obtain the following:

Corollary 2.7 . If $F_{2}(X)$ has pointwise countable type, then the derived set $X^{\prime}$ is compact.

LEMMA 2.8. If $F_{4}(X)$ is of pointwise countable type and $X$ is non-discrete, then $X$ is pseudocompact.

Proof: From the hypothesis that $F_{4}(X)$ is of pointwise countable type, it follows that the space $X$ itself is of pointwise countable type. We claim that $X$ contains an 
infinite compact set $C$. Indeed, using the hypothesis that $X$ is non-discrete, let $y$ be a non-isolated point in $X$ and let $K$ be a compact set containing $y$ such that $X$ has a countable local base at $K$. If $K$ is infinite, we simply take $C=K$. If $K$ is finite, it follows that $X$ has a countable local base at the point $y$, and the fact that $y$ is non-isolated in $X$ implies that there is a non-trivial sequence in $X$ converging to $y$, and we may then take $C$ to consist of the terms of the sequence together with $y$, and the claim is proved.

We now adapt an argument borrowed from the proof of of [15, Theorem 3.5] to show that $X$ must be pseudocompact. Let us assume that $X$ is not pseudocompact. Then we can find a countably infinite discrete family of open sets $\left\{U_{n}: n \in \omega\right\}$ in $X$. We observe that the family of closures $\left\{\overline{U_{n}}: n \in \omega\right\}$ is also discrete, and that $\bigcup_{n \in \omega} \overline{U_{n}}$ is closed in $X$. Now the discreteness of the family $\left\{\overline{U_{n}}: n \in \omega\right\}$ implies that the compact set $C$ can intersect at most a finite number of the sets $\overline{U_{n}}$, and so, by omitting any such sets from the family and re-indexing, we may assume without loss of generality that $C \cap \bigcup_{n \in \omega} \overline{U_{n}}=\emptyset$. It is now clear that the family $\{C\} \cup\left\{U_{n}: n \in \omega\right\}$ is discrete in $X$.

If we now pick $x_{n} \in U_{n}$ for each $n$, then the set $Z=C \cup\left\{x_{n}: n \in \omega\right\}$ is closed, $\sigma$-compact and $P$-embedded in $X$, and therefore, by Theorem 1.7, the subgroup $F(Z, X)$ of $F(X)$ generated by $Z$ is naturally topologically isomorphic to the free topological group $F(Z)$. Moreover, $Z$ is clearly a $k_{\omega}$-space, and it follows by [9] that $F(Z)$ is also a $k_{\omega}$-space.

Since $C$ is an infinite compact set, there exists a non-isolated point $x \in C$. Following [15, Theorem 3.5], we now write $Y=\bigcup_{n \in \omega} C_{n}$, where $C_{n}=x_{n}^{-1} x^{-1} C x_{n}$ for each $n \in \omega$. Then $Y \subseteq F_{4}(Z) \subseteq F_{4}(X)$, and a straightforward argument shows both that $Y$ is closed in $F_{4}(Z)$ and that $Y$ has the quotient topology under the obvious mapping from $\bigoplus_{n \in \omega} C_{n}$ onto $Y=\bigcup_{n \in \omega} C_{n}$. Now the first of these conclusions implies immediately that $Y$ is of pointwise countable type, and we claim that the second implies that it is not. Indeed, the second conclusion implies that any compact subset of $Y$ lies in a finite union $\bigcup_{n \leqslant N} C_{n}$ for some $N \in \omega$, and then a standard diagonal argument using the fact that $x$ is non-isolated in $C$ shows that $Y$ is not of countable type at the point $e \in Y$. This contradiction shows that $X$ must be pseudocompact, as claimed.

We are now in position to prove our first main result on local compactness and pointwise countable type in subspaces of free topological groups.

THEOREM 2.9. The following conditions are equivalent for a space $X$ :

(a) $F_{4}(X)$ is a space of pointwise countable type;

(b) $F_{4}(X)$ is locally compact;

(c) $F_{n}(X)$ is a space of pointwise countable type for each $n \in \omega$;

(d) $F_{n}(X)$ is locally compact for each $n \in \omega$;

(e) the space $X$ is either compact or discrete. 
Proof: It is clear that (b) $\Rightarrow(a),(d) \Rightarrow(c) \Rightarrow(a)$, and that (e) implies each of the conditions (a)-(d). Finally, if $F_{4}(X)$ has pointwise countable type and $X$ is not discrete, then $X$ is pseudocompact by Lemma 2.8. In particular, $X$ is a closed bounded set in itself, so Lemma 2.6 implies that $X$ is compact. This gives the implication (a) $\Rightarrow$ (e). Hence the five conditions are equivalent, as claimed.

Our next step is to characterise the spaces $X$ such that the subspace $A_{n}(X)$ of $A(X)$ is locally compact for each $n \in \omega$. Again, we start with several auxiliary results.

LEMMA 2.10. Let $X$ be an arbitrary space and $\varphi: F(X) \rightarrow A(X)$ be the canonical homomorphism of $F(X)$ onto $A(X)$. Then the restriction $\varphi_{2}=\varphi \uparrow_{F_{2}(X)}$ is a perfect mapping of $F_{2}(X)$ onto $A_{2}(X)$.

Proof: It is clear that $\left|\varphi_{2}^{\leftarrow}(y)\right| \leqslant 2$ for each element $y \in A_{2}(X)$, so the fibres of $\varphi_{2}$ are compact. In addition, the set $Y=X \cup X^{-1}$ is clopen in $F_{2}(X), Y_{a}=X \cup(-X)$ is clopen in $A_{2}(X), \varphi_{2}^{\leftarrow}\left(Y_{a}\right)=Y$ and the restriction of $\varphi_{2}$ to $Y$ is a homeomorphism of $Y$ onto $Y_{a}$. Hence $\varphi_{2}$ is closed at every point of $Y_{a}$.

Let us show that $\varphi_{2}$ is closed at every point $g \in A_{2}(X)$ of length 2 . Let $g=\varepsilon_{1} x_{1}+\varepsilon_{2} x_{2} \neq 0$, where $x_{1}, x_{2} \in X$ and $\varepsilon_{1}, \varepsilon_{2}= \pm 1$. Then $\varphi_{2}^{\leftarrow}(g)=\left\{x_{1}^{\varepsilon_{1}} x_{2}^{\varepsilon_{2}}, x_{2}^{\varepsilon_{2}} x_{1}^{\varepsilon_{1}}\right\}$. If $O$ is an open set in $F_{2}(X)$ containing $\varphi_{2}^{\leftarrow}(g)$, then, by the continuity of the multiplication in $F(X)$, there exist open neighbourhoods $U_{1}$ and $U_{2}$ of $x_{1}$ and $x_{2}$, respectively, in $X$ such that $U_{1}^{\varepsilon_{1}} U_{2}^{\varepsilon_{2}} \cup U_{2}^{\varepsilon_{2}} U_{1}^{\varepsilon_{1}} \subseteq O$. In addition, if $\varepsilon_{1}+\varepsilon_{2}=0$, then $x_{1} \neq x_{2}$, so the sets $U_{1}$ and $U_{2}$ can be chosen to be disjoint. By Proposition 1.5, the set $V=\varepsilon_{1} U_{1}+\varepsilon_{2} U_{2}$ is an open neighbourhood of $g$ in $A_{2}(X)$, and one easily verifies that $\varphi_{2}^{\leftarrow}(V)=U_{1}^{\varepsilon_{1}} U_{2}^{\varepsilon_{2}} \cup U_{2}^{\varepsilon_{2}} U_{1}^{\varepsilon_{1}} \subseteq O$. So the mapping $\varphi_{2}$ is closed at every point of length 2 in $A_{2}(X)$.

Let $e$ be the neutral element of $F(X)$. It is clear that $\varphi_{2}^{\leftarrow}(0)=\{e\}$, where 0 denotes the neutral element of $A(X)$. If $O$ is a neighbourhood of $e$ in $F_{2}(X)$, then, by Theorem 1.1, there exists a continuous pseudometric $d$ on $X$ such that $O_{2}(d) \subseteq O$. The set $O_{2}^{a}(d)=\{x-y: x, y \in X, d(x, y)<1\}$ is an open neighbourhood of 0 in $A_{2}(X)$ by Corollary 1.3 and, clearly, we have $\varphi_{2}^{\leftarrow}\left(O_{2}^{a}(d)\right)=O_{2}(d)$. This proves that $\varphi_{2}$ is closed at the point $0 \in A_{2}(X)$. Therefore, $\varphi_{2}$ is a closed mapping with compact fibres, and so $\varphi_{2}$ is perfect.

It is worth mentioning that Lemma 2.10 cannot be generalised to $n=3$. Indeed, let $X$ be an arbitrary non-compact space and $a_{0} \in X$ be a fixed point. Clearly, there exists an open neighbourhood $U$ of $a_{0}$ in $X$ such that the complement $Y=X \backslash U$ is non-compact. Denote by $\varphi_{3}$ the restriction of the canonical homomorphism $\varphi: F(X) \rightarrow A(X)$ to $F_{3}(X)$. Then $\varphi_{3}^{-1}\left(a_{0}\right)$ contains the closed set $\left\{x a_{0} x^{-1}: x \in Y\right\}$, which is homeomorphic to the non-compact space $Y$. Therefore, the fibre $\varphi_{3}^{-1}\left(a_{0}\right)$ is not compact and, hence, $\varphi_{3}$ fails to be a perfect mapping. We have thus shown that $\varphi_{3}$ has compact fibres if and only if $X$ is compact.

LEMMA 2.11. Let $X=Y \oplus D$ be the topological sum of a space $Y$ and a discrete 
space $D$. Then:

(a) $F_{2}(X)$ is locally compact if and only if $F_{2}(Y)$ is locally compact;

(b) for every $n \in \omega, A_{n}(X)$ is locally compact if and only if $A_{n}(Y)$ is locally compact.

Proof: (a) It is clear that $Y$ is a retract of $X$, so the closed subgroup $F(Y, X)$ of $F(X)$ is topologically isomorphic to the group $F(Y)$ by Proposition 1.8. In particular, $F_{2}(Y)$ can be identified with the closed subspace $F_{2}(Y, X)=F(Y, X) \cap F_{2}(X)$ of $F_{2}(X)$. Therefore, the local compactness of $F_{2}(X)$ implies that of $F_{2}(Y)$.

Conversely, suppose that the space $F_{2}(Y)$ is locally compact. Since $Y$ is closed in $F(Y)$ and $Y \subseteq F_{1}(Y) \subseteq F_{2}(Y)$, the spaces $Y$ and $X=Y \oplus D$ must be locally compact. Therefore, it follows from Proposition 1.5 that $F_{2}(X)$ is locally compact at every element of length 2. It is also clear that the subspace $X \cup X^{-1} \cong X \oplus X$ of $F_{2}(X)$ is clopen in $F_{2}(X)$, so $F_{2}(X)$ is locally compact at all elements of length 1 . It remains to verify the local compactness of $F_{2}(X)$ at the identity $e$.

By our assumption, there exists an open neighbourhood $O$ of the identity in $F_{2}(Y)$ with compact closure. Apply Theorem 1.1 to find a continuous pseudometric $d$ on $Y$ such that

$$
O_{2}(d)=\left\{x^{\varepsilon} y^{-\varepsilon}: x, y \in Y, d(x, y)<1, \varepsilon= \pm 1\right\} \subseteq O .
$$

Clearly, the closure of $O_{2}(d)$ in $F_{2}(Y)$ is also compact. Since $X=Y \oplus D$ and $D$ is discrete, we can extend $d$ to a continuous pseudometric $\varrho$ on $X$ such that $\varrho\left(d, d^{\prime}\right)=1$ if $d, d^{\prime}$ are distinct points of $D$ and $\varrho(y, d) \geqslant 1$ for all $y \in Y$ and $d \in D$. Then, identifying $F(Y)$ with the closed subgroup $F(Y, X)$ of $F(X)$, we obviously have $O_{2}(\varrho)=O_{2}(d)$. Hence the space $F_{2}(X)$ is locally compact at $e$ as well.

(b) The group $A(X)$ is topologically isomorphic in the natural way with $A(Y) \times A(D)$ by Proposition 1.6, and if we identify $A(X)$ and $A(Y) \times A(D)$ under this isomorphism, then we have $A_{n}(X) \subseteq A_{n}(Y) \times A_{n}(D)$. Since $A(D)$ and $A_{n}(D)$ are discrete, this implies (b).

The next lemma is almost evident.

Lemma 2.12. Suppose that in a locally compact space $X$, the derived set $X^{\prime}$ is compact. Then $X$ is homeomorphic to the topological sum of a compact space and a discrete one.

Proof: For every point $x \in X^{\prime}$, choose an open neighbourhood $U_{x}$ of $x$ in $X$ such the closure of $U_{x}$ in $X$ is compact. Using the compactness of $X^{\prime}$, we find a finite set $x_{1}, \ldots, x_{n}$ in $X^{\prime}$ such that $X^{\prime} \subseteq \bigcup_{i=1}^{n} U_{x_{i}}$. Let $K$ be the union of the closures of the sets $U_{x_{i}}$. Then $K$ is compact and $X^{\prime} \subseteq K$. In addition, the set $D=X \backslash K$ has no accumulation points in $X^{\prime}$, so $K$ is open in $X$. Therefore, the space $X$ is homeomorphic to $K \oplus D$, as required. 
We now state and prove our second main result.

THEOREM 2.13. The following conditions are equivalent for a space $X$ :

(a) $F_{2}(X)$ is locally compact;

(b) $A_{2}(X)$ is locally compact;

(c) $A_{n}(X)$ is locally compact for each $n \in \omega$;

(d) $X$ is homeomorphic to the topological sum of a compact space and a discrete space.

Proof: Suppose that $F_{2}(X)$ is locally compact. Then $X$ is also locally compact as a closed subspace of $F_{2}(X)$. By Corollary 2.7, the derived set $X^{\prime}$ is compact. Hence Lemma 2.12 gives the implication (a) $\Rightarrow(d)$. Local compactness is both an invariant and an inverse invariant of perfect mappings by [5, Theorems 3.7.21 and 3.7.24], so the equivalence (b) $\Leftrightarrow$ (a) follows from Lemma 2.10. The implication (c) $\Rightarrow$ (b) is trivial. It is also clear that if $Y$ is a compact space, then $A_{n}(Y)$ is compact for each $n \geqslant 1$. Hence (d) $\Rightarrow$ (c) by Lemma 2.11 .

The next result explains the special role of the number 4 in Theorem 2.9.

Proposition 2.14. The local compactness of $F_{3}(X)$ is equivalent to that of $F_{2}(X)$, for every space $X$.

Proof: By Theorem 2.13, the local compactness of $F_{2}(X)$ implies that $X$ is homeomorphic to a disjoint sum $C \oplus D$, where $C$ is compact and $D$ is discrete. Let $Y$ be the discrete space $\{c\} \oplus D$ obtained from $X$ by identifying $C$ to the point $c$. Let $f: X \rightarrow Y$ be the identification mapping, let $\widehat{f}: F(X) \rightarrow F(Y)$ be an extension of $f$ to a continuous homomorphism, and consider the restriction $f_{3}=\widehat{f}_{F_{3}(X)}$ which maps $F_{3}(X)$ onto $F_{3}(Y)$. We claim that $f_{3}$ is a perfect mapping.

Indeed, it is trivial that $f_{3}$ is closed, since its range is discrete. Also, one can verify routinely that the fibres $f_{3}^{\leftarrow}(w)$ are compact for each $w \in F_{3}(Y)$. For example, the set $f_{3}^{\leftarrow}\left(y_{1}^{\varepsilon_{1}} y_{2}^{\varepsilon_{2}} y_{3}^{\varepsilon_{3}}\right)$, where $y_{1}^{\varepsilon_{1}} y_{2}^{\varepsilon_{2}} y_{3}^{\varepsilon_{3}}$ is a reduced word, is clearly singleton if $y_{1}, y_{2}, y_{3} \in D$, and the set $f_{3}^{\leftarrow}\left(c y_{1}^{\varepsilon_{1}} y_{2}^{\varepsilon_{2}}\right)$, where $y_{1}^{\varepsilon_{1}} y_{2}^{\varepsilon_{2}}$ is a reduced word and $y_{1}, y_{2} \in D$, is the compact set $C y_{1}^{\varepsilon_{1}} y_{2}^{\varepsilon_{2}}$. Similarly, the set $f_{3}^{\leftarrow}\left(y^{\varepsilon}\right)$, where $y \in D$, is the compact set $\left\{y^{\varepsilon}\right\} \cup C C^{-1} y^{\varepsilon}$ $\cup C^{-1} C y^{\varepsilon} \cup y^{\varepsilon} C C^{-1} \cup y^{\varepsilon} C^{-1} C$, and one can perform similar checks for words in $F_{3}(Y)$ of all the other possible forms.

We conclude that $f_{3}$ is indeed a perfect mapping. Finally, since local compactness is an inverse invariant of perfect mappings, by [5, Theorem 3.7.24], we conclude that $F_{3}(X)$ is locally compact, as required.

We cannot extend the above to say that the restriction $f_{4}=\left.\widehat{f}\right|_{F_{4}(X)}$ which maps $F_{4}(X)$ onto $F_{4}(Y)$ is in general perfect, even for very simple perfect mappings. This follows from the next fact:

Proposition 2.15. Let $f: X \rightarrow Y$ be a continuous onto mapping. The 
mapping $f_{4}: F_{4}(X) \rightarrow F_{4}(Y)$ is perfect if and only if either $f$ is a homeomorphism or $X$ is compact.

Proof: If $f$ is a homeomorphism, then its extension $\widehat{f}: F(X) \rightarrow F(Y)$ is a topological isomorphism and the mapping $f_{4}=\widehat{f}_{F_{4}(X)}$ is a homeomorphism between $F_{4}(X)$ and $F_{4}(Y)$. If $X$ is compact, then $F_{4}(X)$ is also compact, so $f_{4}$ is again perfect.

Conversely, suppose that the mapping $f_{4}$ is perfect. Then its restriction $f=\left.f_{4}\right|_{X}$ to the closed subspace $X$ of $F_{4}(X)$ is also perfect. So, if $f$ is one-to-one, then $f$ is a homeomorphism, and we are done. Suppose, therefore, that $f$ has a non-trivial fibre. Choose distinct points $a, b \in X$ such that $f(a)=f(b)$. If $X$ is not compact, there exist open neighbourhoods $U$ and $V$ of $a$ and $b$ in $X$, respectively, such that the complement $Y=X \backslash(U \cup V)$ is not compact. The set

$$
P=\left\{x a b^{-1} x^{-1}: x \in Y\right\}
$$

is closed in $F_{4}(X)$ and is contained in $f_{4}^{\leftarrow}(e)$, where $e$ is the identity of $F(Y)$. In addition, $P$ is homeomorphic to the non-compact space $Y$, so the fibre $f_{4}^{\leftarrow}(e)$ is not compact. Thus the mapping $f_{4}$ is not perfect.

\section{MORE ON POINTWISE COUNTABLE TYPE}

Our next step is to characterise, in a manner analogous to Theorem 2.13 , the spaces $X$ such that $F_{2}(X)$ or $A_{2}(X)$ is of pointwise countable type. We shall see in Theorem 3.5 that, in both cases, this is equivalent to the existence of a compact set $C$ of countable character in $X$ such that $X^{\prime} \subseteq C$.

Let $X$ be a space and $\Delta_{X}$ be the diagonal in $X^{2}$. Denote by $\mathcal{U}_{X}$ the finest uniformity on $X$ compatible with the topology of $X$. For every $U \in \mathcal{U}$, put

$$
O_{2}(U)=\left\{x^{\varepsilon} y^{-\varepsilon}:(x, y) \in U, \varepsilon= \pm 1\right\} .
$$

The lemma below is a reformulation of Theorem 1.1.

Lemma 3.1. The sets $O_{2}(U)$, with $U \in \mathcal{U}_{X}$, form a base at the neutral element $e$ for the space $F_{2}(X)$.

The next fact is a simple part of Theorem 3.3.

Proposition 3.2. Let $C$ be a compact subset of a space $X$ such that $X^{\prime} \subseteq C$ and $\chi(C, X) \leqslant \omega$. Then $F_{2}(X)$ has pointwise countable type.

Proof: If $X$ is discrete, then the spaces $F(X)$ and $F_{2}(X)$ are discrete. Suppose, therefore, that $X^{\prime} \neq \emptyset$. Our assumptions about $X$ imply that $X$ is of pointwise countable type. Hence the space $X^{2}$ also has pointwise countable type. Let $e$ denote the neutral element of the group $F(X)$. Since $X \oplus X^{-1} \cong F_{1}(X) \backslash\{e\}$ is clopen in $F_{2}(X)$ and the space $F_{2}(X) \backslash F_{1}(X)$ is locally homeomorphic to $X^{2}$, it suffices to find a compact set in 
$F_{2}(X)$ which contains $e$ and has countable character in $F_{2}(X)$. Put $K=C C^{-1} \cup C^{-1} C$. Let us verify that the compact set $K \subseteq F_{2}(X)$ is as required. It is clear that $e \in K$. Since $\chi(C, X) \leqslant \omega$, the space $X$ has a countable base $\left\{U_{n}: n \in \omega\right\}$ at $C$. For every $n \in \omega$, let $V_{n}=U_{n} U_{n}^{-1} \cup U_{n}^{-1} U_{n}$. We claim that the family $\left\{V_{n}: n \in \omega\right\}$ is a base for $F_{2}(X)$ at $K$.

Indeed, let $O$ be an open neighbourhood of $K$ in $F_{2}(X)$. Since $C$ is compact, there exists an open neighbourhood $U$ of $C$ in $X$ such that $U U^{-1} \subseteq O$ and $U^{-1} U \subseteq O$. Choose $n \in \omega$ with $U_{n} \subseteq U$. Then $V_{n} \subseteq O$, so it remains to show that $V_{n}$ is open in $F_{2}(X)$. Clearly, the set $W_{n}=\left(U_{n} \times U_{n}\right) \cup \Delta_{X}$ is open in $X^{2}$. Since $X$ is paracompact by Lemma 2.3 , we have $W_{n} \in \mathcal{U}_{X}$ for all $n \in \omega$, and one easily verifies that $O_{2}\left(W_{n}\right)=V_{n}$. This implies our claim and shows that $F_{2}(X)$ is of pointwise countable type.

THEOREM 3.3. The following conditions are equivalent for a space $X$ :

(a) $F_{2}(X)$ has pointwise countable type;

(b) there exists a compact set $C$ in $X$ such that $X^{\prime} \subseteq C$ and $\chi(C, X) \leqslant \omega$.

Proof: The implication (b) $\Rightarrow$ (a) follows from Proposition 3.2. Conversely, suppose that $F_{2}(X)$ is of pointwise countable type and take a compact set $K \subseteq F_{2}(X)$ which contains the identity $e$ of $F(X)$ and satisfies $\chi\left(K, F_{2}(X)\right) \leqslant \omega$. By the Nummela-Pestov theorem, $F(X)$ is identified with the subgroup $F(X, \mu X)$ of $F(\mu X)$. Then $F_{2}(X)$ is identified with the subspace $F_{2}(\mu X) \cap F(X)$ of $F_{2}(\mu X)$. Observe that $F_{2}(X)$ is dense in $F_{2}(\mu X)$ since $X$ is dense in $\mu X$. Hence Lemma 2.2 implies that $\chi\left(K, F_{2}(\mu X)\right) \leqslant \omega$. Let $\left\{U_{n}: n \in \omega\right\}$ be a countable base for $F_{2}(\mu X)$ at $K$. By Theorem 1.1, there exists, for every $n \in \omega$, a continuous pseudometric $d_{n}$ on $\mu X$ such that $O_{2}\left(d_{n}\right) \subseteq U_{n}$. We can assume without loss of generality that each $d_{n}$ is bounded by 1 . Then $d=\sum_{n=0}^{\infty} 2^{-n} d_{n}$ is a continuous pseudometric on $\mu X$ and one easily verifies that the set

$$
P=\left\{x^{-1} y: x, y \in \mu X, d(x, y)=0\right\}
$$

satisfies $P \subseteq \bigcap_{n \in \omega} O_{2}\left(d_{n}\right) \subseteq \bigcap_{n \in \omega} U_{n}=K$. In particular, $P \subseteq F_{2}(X)$. The set $P$ is closed in $F_{2}(\mu X)$ since the pseudometric $d$ is continuous. Hence $P$ is compact as a closed subset of $K$.

Consider the continuous mapping $i_{2}: X^{2} \rightarrow F_{2}(X), i_{2}(x, y)=x^{-1} y$ for $x, y \in X$. Since $X^{\prime}$ is compact by Corollary 2.7, it follows from Lemma 2.3 that the space $X$ is paracompact. So [22, Proposition 4.8] implies that $i_{2}$ is a closed mapping. Observe that the restriction of $i_{2}$ to the closed subspace

$$
Z=X^{2} \backslash\left\{(x, x): x \in X \backslash X^{\prime}\right\}
$$

of $X^{2}$ has compact fibres. In fact, the unique non-trivial fibre of $i_{2} \mid z$ can only be $i_{2}^{+}(e)$ $=\left\{(x, x): x \in X^{\prime}\right\}$. Therefore, this restriction is a perfect mapping (onto its image). 
Since $P$ is compact,

$$
L=Z \cap i_{2}^{\leftarrow}(P)=\left\{(x, y) \in X^{2}: d(x, y)=0\right\} \backslash\left\{(x, x): x \in X \backslash X^{\prime}\right\}
$$

is a compact subset of $Z$. Let $\pi: X^{2} \rightarrow X$ be the projection to the second factor. Then $C=\pi(L)$ is a compact subset of $X$ and, clearly, $X^{\prime} \subseteq C$.

It remains to verify that $\chi(C, X) \leqslant \omega$. For every $n \in \omega$, put

$$
V_{n}=\left\{x \in X: d(x, C)<2^{-n}\right\}
$$

Then $\gamma=\left\{V_{n}: n \in \omega\right\}$ is a countable family of open neighbourhoods of $C$ in $X$, and we claim that $\gamma$ is a base for $X$ at $C$.

First, we verify that $d(y, C)>0$ for each $y \in X \backslash C$. Indeed, if $y \in X \backslash C$ and $d(x, y)=0$ for some $x \in C$, then $(x, y) \in L$ and $y \in C$, which is a contradiction. Hence $d(x, y)>0$ for each $x \in C$. Since the set $C$ is compact, we conclude that $d(y, C)>0$. Observe that this implies the equality $C=\cap \gamma$.

Suppose to the contrary that $\gamma$ fails to be a base for $X$ at $C$. Then there exists an open set $O$ in $X$ such that $C \subseteq O$ and $V_{n} \backslash O \neq \emptyset$ for each $n \in \omega$. Therefore, we can choose two sequences $\left\{x_{n}: n \in \omega\right\}$ and $\left\{y_{n}: n \in \omega\right\}$ such that $x_{n} \in C, y_{n} \in V_{n} \backslash O$ and $d\left(x_{n}, y_{n}\right)<2^{-n}$ for each $n \in \omega$. Then our definition of $d$ as an infinite linear combination of the pseudometrics $d_{n}$ implies that $d_{n}\left(x_{n}, y_{n}\right)<1$, whence it follows that $g_{n}=x_{n}^{-1} y_{n} \in U_{n}$. Clearly, $x_{n} \neq y_{n}$, so $g_{n} \neq e$ for each $n \in \omega$. From our choice of the points $y_{n}$ it follows that the positive distances $d\left(y_{n}, C\right)$ tend to zero, so the set $\left\{y_{n}: n \in \omega\right\}$ is infinite. Since the sets $U_{n}$ form a base for $F_{2}(X)$ at the compact set $K$, the infinite set $S=\left\{g_{n}: n \in \omega\right\}$ has an accumulation point in $K$. On the other hand, the set $T=\left\{\left(x_{n}, y_{n}\right): n \in \omega\right\}$ is closed and discrete in $X^{2}$. Indeed, since $X^{\prime} \subseteq C \subseteq O$, the set $X \backslash O$ is closed and discrete in $X$. Hence the set $Y=\left\{y_{n}: n \in \omega\right\} \subseteq X \backslash O$ is also closed and discrete in $X$. Since $\pi(T)=Y$, so is $T$ in $X^{2}$. Finally, the mapping $i_{2}$ is closed and $i_{2}(T)=S$, so that $S$ is infinite, closed and discrete in $F_{2}(X)$. This contradiction shows that (a) implies (b), as claimed.

As in Theorems 2.9 and 2.13, it is natural to ask for a characterisation of the spaces $X$ such that each $A_{n}(X)$ has pointwise countable type. It turns out that the latter property is equivalent to that for the single number $n=2$. Our proof of this fact requires the following proposition:

Proposition 3.4. Let $C$ be a compact subset of a space $X$ such that $X^{\prime} \subseteq C$ and $\chi(C, X) \leqslant \omega$. Then $A_{n}(X)$ has point-countable type for every $n \in \omega$.

Proof: We show first that $A_{2 n}(X)$ has countable type at the identity 0 for each integer $n$. Fix $n \in \omega$. If $X$ is discrete, there is nothing to prove, so we assume that $X^{\prime} \neq \emptyset$. Let $K=n C-n C$, and note that $K$ is a compact subset of $A_{2 n}(X)$ which contains 0. Fix a countable base $\left\{U_{k}: k \in \omega\right\}$ for the space $X$ at $C$, and for every $k \in \omega$ 
let $V_{k}=n U_{k}-n U_{k}$. Clearly, $K \subseteq V_{k} \subseteq A_{2 n}(X)$ for each $k$, and we claim that the family $\left\{V_{k}: k \in \omega\right\}$ is a base for $A_{2 n}(X)$ at $K$.

Let $O$ be an open neighbourhood of $K$ in $A_{2 n}(X)$. Since $C$ is compact in $X$, there exists an open neighbourhood $U$ of $C$ in $X$ such that $n U-n U \subseteq O$. If we choose $k \in \omega$ with $U_{k} \subseteq U$, then clearly $V_{k} \subseteq O$. It is easy to see that the set $W_{k}=\left(U_{k} \times U_{k}\right) \cup \Delta_{X}$ is open in $X^{2}$, where $\Delta_{X}$ denotes the diagonal in $X^{2}$. Then since $X$ is paracompact, by Lemma 2.3, we have $W_{k} \in \mathcal{U}_{X}$. Let $s: \omega \rightarrow \mathcal{U}_{X}$ be a constant function, $s(n)=W_{k}$ for each $n \in \omega$. It is easy to see that $O(s) \cap A_{2 n}=V_{k}$, and it follows from Theorem 1.4 that $V_{k}$ is open in $A_{2 n}(X)$. We conclude that, as claimed, $A_{2 n}(X)$ has countable type at 0.

Now let $g$ be an arbitrary element of $A_{n}(X)$ for a fixed $n \in \omega$. Also, let $K$ be a compact subset of $A_{2 n}(X)$ which contains 0 and at which $A_{2 n}(X)$ has a countable base $\left\{V_{k}: k \in \omega\right\}$. Write $C=(K+g) \cap A_{n}(X)$, and note that $C$ is a compact subset of $A_{n}(X)$ containing $g$. We claim that the collection $\left\{W_{k}: k \in \omega\right\}$, where $W_{k}=\left(V_{k}+g\right) \cap A_{n}(X)$ for each $k \in \omega$, forms a base for $A_{n}(X)$ at $C$.

Indeed, since $V_{k}$ is open in $A_{2 n}(X)$, the set $V_{k}+g$ is open in $A_{2 n}(X)+g$, and since $A_{n}(X) \subseteq A_{2 n}(X)+g$, we see that $W_{k}=\left(V_{k}+g\right) \cap A_{n}(X)$ is open in $\left(A_{2 n}(X)+g\right)$ $\cap A_{n}(X)=A_{n}(X)$. Also, if $C \subseteq V \subseteq A_{n}(X)$, where $V$ is an open set in $A_{n}(X)$, let $W=V \cup\left(A(X) \backslash A_{n}(X)\right)$, and note that $W$ is an open set in $A(X)$. Now $C=(K+g)$ $\cap A_{n}(X) \subseteq V$ implies $K+g \subseteq V \cup\left(A(X) \backslash A_{n}(X)\right)=W$, and so $K \subseteq W-g$. Therefore, since $W-g$ is an open set in $A(X)$, there exists $k \in \omega$ such that $K \subseteq V_{k} \subseteq W-g$, and we have

$$
(K+g) \cap A_{n}(X) \subseteq\left(V_{k}+g\right) \cap A_{n}(X) \subseteq W \cap A_{n}(X),
$$

that is, $C \subseteq W_{k} \subseteq V$. We conclude that $A_{n}(X)$ is of pointwise countable type, as required.

We now present our third main result.

THEOREM 3.5. The following conditions are equivalent for a space $X$ :

(a) $F_{2}(X)$ has pointwise countable type;

(b) $A_{2}(X)$ has pointwise countable type;

(c) $A_{n}(X)$ has pointwise countable type for each $n \in \omega$;

(d) $X$ contains a compact set $C$ such that $X^{\prime} \subseteq C$ and $\chi(C, X) \leqslant \omega$.

Proof: The implication (d) $\Rightarrow$ (c) is given by Proposition 3.4, while the implication (c) $\Rightarrow$ (b) is trivial. The restriction of the canonical homomorphism $\varphi: F(X) \rightarrow A(X)$ to $F_{2}(X)$ is a perfect mapping onto $A_{2}(X)$ by Lemma 2.10 , and having pointwise countable type is an inverse invariant of perfect mappings. (Indeed, if $f: X \rightarrow Y$ is a perfect onto mapping and $C$ is a compact set of countable character in $Y$, then $f^{-1}(C)$ is again a compact set of countable character in $X$.) Hence (b) implies (a). Finally, the implication (a) $\Rightarrow$ (d) is given by Theorem 3.3 . 
We shall show in Proposition 3.7 that, as in the case of local compactness, $F_{3}(X)$ has pointwise countable type if and only if $F_{2}(X)$ has pointwise countable type. Our proof is based on the following auxiliary fact.

LEMMA 3.6. Let $U$ be an open set in a space $X$ such that $X^{\prime} \subseteq U$. Then the set $W=U U U^{-1} \cup U U^{-1} U \cup U^{-1} U U$ is open in $F_{3}(X)$.

Proof: Clearly, $U$ and $V=X \backslash U$ are clopen disjoint sets and $V$ is discrete, so we can take a continuous pseudometric $d$ on $X$ such that $d\left(x, x^{\prime}\right)=0$ for all $x, x^{\prime} \in U$, $d\left(x, x^{\prime}\right)=1$ for all $x, x^{\prime} \in V$, and $d(x, y)=1$ for all $x \in U$ and $y \in V$. Let $\hat{d}$ be Graev's extension of $d$ to a continuous invariant pseudometric on $F(X)$ such that $\widehat{d}\left(x^{-1}, y\right)$ $=\widehat{d}\left(x, y^{-1}\right)=\widehat{d}(x y, e) \geqslant 1$ for all $x, y \in X$, where $e$ is the identity of $F(X)$. Since $\widehat{d}$ is continuous, the set

$$
O=\{g \in F(X): \widehat{d}(g, e)<1\}
$$

is an open neighbourhood of $e$ in $F(X)$. Hence $G=U O \cup O U$ is also open in $F(X)$, and we claim that

$$
G \cap F_{3}(X)=W .
$$

Indeed, we have $U U^{-1} \cup U^{-1} U \subseteq O$, so the inclusion $W \subseteq G \cap F_{3}(X)$ is immediate. Conversely, let $x \in U$ and $g \in O$ be arbitrary elements such that $h=x g \in F_{3}(X)$, and observe that $g$ must have even length $\leqslant 4$. If $g=e$, then $h=x \in U \subseteq W$. If the length of $g$ equals 2, then $g=x^{\varepsilon} y^{-\varepsilon}$ for some $x, y \in X$ and $\varepsilon= \pm 1$. It follows from $g \in O$ that $x, y \in U$, so $h=x g \in U U^{\varepsilon} U^{-\varepsilon} \subseteq W$. The last possibility is that $g$ has length 4, so that $h=x g=x\left(x^{-1} y z t\right)=y z t$, where $x \in U$ and $y, z, t \in X \cup X^{-1}$. Since $g=x^{-1} y z t \in O$, we have $\widehat{d}(g, e)<1$. Let us consider the following two cases.

(a) $\widehat{d}(g, e)=\hat{d}\left(x^{-1} y, e\right)+\widehat{d}(z t, e)=\widehat{d}(y, x)+\widehat{d}\left(t, z^{-1}\right)<1$. Then $\hat{d}(y, x)<1$, so $y \in X$ and $x, y \in U$. In addition, we have $\widehat{d}\left(t, z^{-1}\right)<1$, whence it follows that $z^{-1}, t \in X^{\varepsilon}$ for some $\varepsilon= \pm 1$ and, by the choice of $d$, this gives $z^{-1}, t \in U^{\varepsilon}$. Thus $h=y z t \in U U^{-\varepsilon} U^{\varepsilon} \subseteq W$.

(b) $\hat{d}(g, e)=\widehat{d}\left(x^{-1} t, e\right)+\widehat{d}(y z, e)=\widehat{d}(t, x)+\hat{d}\left(z, y^{-1}\right)<1$. Then $\hat{d}(t, x)<1$, so $t \in X$ and $x, t \in U$. Similarly, from $\widehat{d}\left(z, y^{-1}\right)<1$ it follows that $z, y^{-1} \in X^{\varepsilon}$ for some $\varepsilon= \pm 1$, so $z, y^{-1} \in U^{\varepsilon}$, by our choice of $d$. We conclude that $h=y z t \in U^{-\varepsilon} U^{\varepsilon} U \subseteq W$.

Combining (a) and (b), we deduce the inclusion $U O \cap F_{3}(X) \subseteq W$. A similar argument gives the inclusion $O U \cap F_{3}(X) \subseteq W$, so we finally have that $G \cap F_{3}(X) \subseteq W$. This proves the equality $(*)$ and implies that $W$ is open in $F_{3}(X)$.

Proposition 3.7. For every space $X, F_{3}(X)$ is of pointwise countable type if and only if so is $F_{2}(X)$.

Proof: Since $F_{2}(X)$ is a closed subspace of $F_{3}(X)$, it suffices to prove the sufficiency. Suppose that $F_{2}(X)$ has pointwise countable type. Then, by Theorem 3.3, there 
exists a compact set $C$ of countable character in $X$ such that $X^{\prime} \subseteq C$. Observe that $P=\left(F_{2}(X) \backslash F_{1}(X)\right) \cup\{e\}$ is a clopen subset of $F_{3}(X)$ (see $[2,8]$ ), and $P$ is closed in $F_{2}(X)$. Hence $F_{3}(X)$ has countable type at the identity $e$ and at each element of length 2. Clearly, $X$ is of pointwise countable type as a closed subspace of $F_{2}(X)$, and so are $F_{1}(X) \cong X \oplus\{e\} \oplus X^{-1}$ and $F_{1}(X)^{3}$. Since the spaces $F_{3}(X) \backslash F_{2}(X)$ and $F_{1}(X)^{3}$ are locally homeomorphic by Proposition $1.5, F_{3}(X)$ is of countable type at all elements of length 3.

It remains to show that, for every element $x \in F_{3}(X)$ of length 1 , there exists a compact set $K$ of countable character in $F_{3}(X)$ such that $x \in K$. Since taking inverses is a homeomorphism of $F_{3}(X)$ onto itself, we can assume that $x \in X$. If $x \in C$, put $K=C C C^{-1} \cup C C^{-1} C \cup C^{-1} C C$. It is clear that $K$ is a compact subset of $F_{3}(X)$ and $x \in C \subseteq K$. By our choice of $C$, there exists a countable base $\left\{U_{n}: n \in \omega\right\}$ for $X$ at $C$. For every $n \in \omega$, let $W_{n}=U_{n} U_{n} U_{n}^{-1} \cup U_{n} U_{n}^{-1} U_{n} \cup U_{n}^{-1} U_{n} U_{n}$. We claim that $\gamma=\left\{W_{n}: n \in \omega\right\}$ is a countable base for $F_{3}(X)$ at $K$.

Indeed, since $X^{\prime} \subseteq C \subseteq U_{n}$ for each $n$, Lemma 3.6 implies that each $W_{n}$ is open in $F_{3}(X)$. It is also clear that $K \subseteq W_{n}$ for each $n \in \omega$. Let $O$ be an arbitrary open neighbourhood of $K$ in $F_{3}(X)$. Take a neighbourhood $W$ of $K$ in $F(X)$ such that $W \cap F_{3}(X)=O$. Since the set $C$ is compact, we can find an open neighbourhood $V$ of $C$ in $F(X)$ such that $V V V^{-1} \cup V V^{-1} V \cup V^{-1} V V \subseteq W$. Choose $n \in \omega$ such that $U_{n} \subseteq V \cap X$. We then have $K \subseteq W_{n} \subseteq W \cap F_{3}(X)=O$, which implies the claim.

Finally, if $x \in X \backslash C$, then $x$ is an isolated point of $X$. The compact set $C_{x}=C \cup\{x\}$ also has countable character in $X$, so one can apply the above argument to the set $K_{x}=C_{x} C_{x} C_{x}^{-1} \cup C_{x} C_{x}^{-1} C_{x} \cup C_{x}^{-1} C_{x} C_{x}$ and show that $K_{x}$ has countable character in $F_{3}(X)$. This finishes the proof.

Cech-completeness occupies a place between local compactness and pointwise countable type. In a sense, Čech-completeness is a kind of uniform pointwise countable type. Let us show that, for spaces of the form $A_{2}(X)$ or $F_{2}(X)$, Cech-completeness and pointwise countable type coincide.

We recall that a continuous pseudometric $d$ on a space $Y$ is called complete if, for every Cauchy sequence $\left\{y_{n}: n \in \omega\right\} \subseteq Y$ with respect to $d$, there exists a point $y \in Y$ such that $d\left(y_{n}, y\right) \rightarrow 0$ when $n \rightarrow \infty$.

THEOREM 3.8. The following conditions are equivalent for a space $X$ :

(a) $A_{2}(X)$ is Cech-complete;

(b) $F_{2}(X)$ is Cech-complete;

(c) $A_{2}(X)$ has pointwise countable type;

(d) $F_{2}(X)$ has pointwise countable type.

Proof: Since the natural mapping $\varphi: F_{2}(X) \rightarrow A_{2}(X)$ is perfect (see Lemma 2.10) and Čech-completeness is both an invariant and an inverse invariant of perfect mappings 
by [5, Theorem 3.9.10], we have (a) $\Leftrightarrow$ (b). It also follows from Theorem 3.5 that (c) $\Leftrightarrow$ (d). The implications (a) $\Rightarrow$ (c) and (b) $\Rightarrow$ (d) are trivial. Therefore, it suffices to show that $(d) \Rightarrow(b)$.

Suppose that the space $F_{2}(X)$ has pointwise countable type. Then, by Theorem 3.3, there exists a compact set $C$ of countable character in $X$ such that $X^{\prime} \subseteq C$. Let $\left\{U_{n}: n \in \omega\right\}$ be a decreasing base for $X$ at $C$. For every $n \in \omega$, choose a continuous pseudometric $d_{n}$ on $X$ such that $d_{n} \leqslant 1, d_{n}(x, y)=0$ for all $x, y \in C, d_{n}(x, y)=1$ if $x, y$ are distinct points in $X \backslash U_{n}$, and $d_{n}(x, y)=1$ for all $x \in U_{n}$ and $y \in X \backslash U_{n}$. Then $d=\sum_{n=0}^{\infty} 2^{-n} d_{n}$ is a continuous pseudometric on $X$ having the following properties:

(i) $d(x, y)=0$ for all $x, y \in C$;

(ii) $d(x, y) \geqslant 2^{-n}$ if $x, y$ are distinct points in $X \backslash U_{n}$;

(iii) $d(x, y) \geqslant 2^{-n}$ for all $x \in U_{n}$ and $y \in X \backslash U_{n}, n \in \omega$.

We claim first that $d$ is a complete pseudometric. Indeed, let $S=\left\{x_{k}: k \in \omega\right\}$ be a Cauchy sequence in $X$ with respect to $d$. If $S \cap U_{n} \neq \emptyset$ for each $n \in \omega$, then $S$ has an accumulation point $y \in C$, so that $S$ converges to $y$ with respect to $d$. Suppose, therefore, that $U_{m} \cap S=\emptyset$ for some $m \in \omega$. Then $d\left(x_{k}, x_{l}\right) \geqslant 2^{-m}$ for distinct $x_{k}, x_{l} \in S$ and, since $S$ is a Cauchy sequence, $S$ is eventually constant. In particular, $S$ converges. This proves our claim.

Let $\widehat{d}$ be Graev's extension of $d$ over $F(X)$ such that $\widehat{d}(x, e)=\widehat{d}\left(x^{-1}, e\right) \geqslant 1$ and $\widehat{d}\left(x, y^{-1}\right)=\widehat{d}(x y, e)=\widehat{d}\left(x^{-1}, y\right) \geqslant 1$ for all $x, y \in X$, where $e$ is the identity of $F(X)$. Denote by $d_{2}$ the restriction of $\widehat{d}$ to $F_{2}(X)$. We claim that $d_{2}$ is a complete pseudometric. Indeed, since $d$ is complete, it follows from our choice of $\widehat{d}$ that $d_{2}$ is complete on $F_{1}(X)$ $\cong X \oplus\{e\} \oplus X^{-1}$. Observe also that $d_{2}(x, g) \geqslant 1$ for every $x \in X \cup X^{-1}$ and $g \in\left(F_{2}(X) \backslash F_{1}(X)\right) \cup\{e\}$. Therefore, it suffices to prove that $d_{2}$ is complete on $P=\left(F_{2}(X) \backslash F_{1}(X)\right) \cup\{e\}$.

Suppose that $T=\left\{g_{n}: n \in \omega\right\}$ is a Cauchy sequence in $P$ with respect to $d_{2}$. We can assume without loss of generality that $d_{2}\left(g_{n}, g_{m}\right)<1$ for all $n, m \in \omega$. Every element $g_{n}$ has the form $g_{n}=x_{n}^{\varepsilon_{n}} y_{n}^{\delta_{n}}$ for some $x_{n}, y_{n} \in X$ and $\varepsilon_{n}, \delta_{n}= \pm 1$. Choose an infinite set $A \subseteq \omega$ and $\varepsilon, \delta= \pm 1$ such that $\varepsilon_{n}=\varepsilon$ and $\delta_{n}=\delta$ for each $n \in A$. By our choice of $A$ and the above assumption, we have $d_{2}\left(g_{n}, g_{m}\right)=d_{2}\left(g_{n} g_{m}^{-1}, e\right)<1$ or, equivalently, $d_{2}\left(x_{n}^{\varepsilon} y_{n}^{\delta} y_{m}^{-\delta} x_{m}^{-\varepsilon}, e\right)<1$ for all $n, m \in \omega$. Hence, for any given pair $n, m \in \omega$, either

$$
d_{2}\left(g_{n}, g_{m}\right)=d\left(x_{n}, x_{m}\right)+d\left(y_{n}, y_{m}\right)
$$

or

$$
d_{2}\left(g_{n}, g_{m}\right)=d_{2}\left(x_{n}, y_{n}\right)+d\left(x_{m}, y_{m}\right) \text { and } \varepsilon+\delta=0 .
$$

Clearly, if $\varepsilon+\delta \neq 0$, then (1) holds for all $n, m \in A$. Consider the set

$$
[A]^{2}=\{\{n, m\}: n, m \in A, n \neq m\}
$$


and define a mapping $f:[A]^{2} \rightarrow\{1,2\}$ by $f(\{n, m\})=1$ if $(1)$ holds for the pair $\{n, m\}$ with $n<m$; otherwise put $f(\{n, m\})=2$. By Ramsey's theorem (see [16, p. 7]), one can find an infinite set $B \subseteq A$ and $i \in\{1,2\}$ such that $f(\{n, m\})=i$ for all distinct $n, m \in B$. If $i=1$, then it follows from (1) that both $S_{1}=\left\{x_{n}: n \in B\right\}$ and $S_{2}=\left\{y_{n}: n \in B\right\}$ are Cauchy sequences with respect to $d$. Since $d$ is complete on $X, S_{1}$ converges to a point $x \in X$ and $S_{2}$ converges to $y \in X$, both with respect to $d$. Hence the sequences $\left\{g_{n}: n \in B\right\}$ and $\left\{g_{n}: n \in \omega\right\}$ converge to the element $g=x^{\varepsilon} y^{\delta}$ with respect to the pseudometric $d_{2}$. Finally, if $i=2$, then (2) implies that $\delta=-\varepsilon$ and $d\left(x_{n}, y_{n}\right) \leqslant d_{2}\left(g_{n}, g_{m}\right)$ for any $n, m \in B$ with $n<m$, so that the distances $d\left(x_{n}, y_{n}\right)$ tend to zero when $n \rightarrow \infty, n \in B$. In its turn, this implies that $d_{2}\left(g_{n}, e\right) \rightarrow 0$ when $n \rightarrow \infty$, $n \in B$. Since $\left\{g_{n}: n \in \omega\right\}$ is a Cauchy sequence, we conclude that $\lim _{n \rightarrow \infty} d_{2}\left(g_{n}, e\right)=0$. This proves that $d_{2}$ is a complete pseudometric.

Consider the equivalence relation $\sim$ on $F_{2}(X)$ defined by $g \sim h$ if and only if $d(g, h)$ $=0$ and let $Y=F_{2}(X) / \sim$ be the quotient set. Let $p: F_{2}(X) \rightarrow Y$ be the natural projection, $p(g)=[g]$ for each $x \in Y$, where $[g]=\left\{h \in F_{2}(X): g \sim h\right\}$. Define a function $\varrho$ on $Y^{2}$ by $\varrho(p(g), p(h))=d_{2}(g, h)$ for all $g, h \in F_{2}(X)$. It is clear that $\varrho$ is a metric on $Y$. Let $\tau$ be the topology on $Y$ generated by $\varrho$. We claim that the mapping $p: F_{2}(X) \rightarrow(Y, \tau)$ is perfect.

It easily follows from our definition of $d_{2}$ that $[g]=\{g\}$ for each $g=x^{\varepsilon} y^{\delta} \in F_{2}(X) \backslash$ $F_{1}(X)$ with $x, y \in X \backslash C$ and $\varepsilon, \delta= \pm 1$. If only one of the points $x, y$ is in $C$, say, $x \in C$, then $[g]=C^{\varepsilon} y^{\delta}$. If both points $x, y$ are in $C$, then $[g]=C^{\varepsilon} C^{\delta}$. Similarly, if $g=x^{\varepsilon}$ with $x \in X$ and $\varepsilon= \pm 1$, then $[g]=\{g\}$ if $x \notin C$, and $[g]=C^{\varepsilon}$ otherwise. Finally, $[e]=C C^{-1} \cup C^{-1} C$. Therefore, the fibres of the mapping $p$ are compact.

It remains to verify that $p$ is a closed mapping. For every integer $n \geqslant 1$ and $y \in Y$, denote by $B(y, 1 / n)$ the open ball with centre at $y$ and radius $1 / n$ with respect to $\varrho$. Let us show that $p$ is closed at the point $a=p(e)$. Suppose that $O$ is a neighbourhood of the fibre $p^{-1}(a)=[e]=C C^{-1} \cup C^{-1} C$ in $F_{2}(X)$. Since $C$ is compact, there exists an open neighbourhood $U$ of $C$ in $X$ such that $U U^{-1} \cup U^{-1} U \subseteq O$. Choose an integer $n \geqslant 1$ such that $U_{n} \subseteq U$. Then $p^{-1}\left(B\left(a, 2^{-n}\right)\right) \subseteq O$. Indeed, suppose that $g \in F_{2}(X)$ and $\varrho(p(g), a)=d_{2}(g, e)<2^{-n}$. Then either $g=e \in O$ or $g=x^{\varepsilon} y^{-\varepsilon} \in F_{2}(X) \backslash F_{1}(X)$, where $\varepsilon= \pm 1$. In the latter case, we have

$$
d_{2}(g, e)=d_{2}\left(x^{\varepsilon} y^{-\varepsilon}, e\right)=d_{2}\left(x^{\varepsilon}, y^{\varepsilon}\right)=d_{2}(x, y)=d(x, y)<2^{-n}
$$

Since $g \neq e$, we necessarily have that $x \neq y$. It follows now from (ii) and (iii) that $x, y \in U_{n}$, so that $g=x^{\varepsilon} y^{-\varepsilon} \in U_{n}^{\varepsilon} U_{n}^{-\varepsilon} \subseteq O$. This proves the inclusion $p^{-1}\left(B\left(a, 2^{-n}\right)\right) \subseteq O$ and, hence, $p$ is closed at the point $a=p(e)$. A similar argument (with certain simplifications) shows that $p$ is closed at any point of $Y \backslash\{a\}$. Therefore, $p$ is a perfect mapping.

Since the pseudometric $d_{2}$ is complete, we conclude that the metric space $(Y, \varrho)$ is 
complete. In particular, the space $(Y, \tau)$ is Čech-complete. Hence $F_{2}(X)$ is Čech-complete as a perfect preimage of a Čech-complete space.

One can modify the argument in the proof of Theorem 3.8 to show that the restriction of the pseudometric $\widehat{d}$ to $F_{3}(X)$ remains complete, so that $F_{3}(X)$ is Čech-complete if and only if so is $F_{2}(X)$.

\section{AlMOST METRISABILITY OF FREE GROUPS}

A topological group $G$ is called almost metrisable if it contains a non-empty compact set of countable character in $G$ [13]. Since topological groups are homogeneous, every almost metrisable group has pointwise countable type, and vice versa. It is clear that all locally compact groups as well as all metrisable groups are almost metrisable. It turns out that the free (Abelian) topological group on a space $X$ is almost metrisable if and only if $X$ is discrete (see Theorem 2.9). Our proof of this fact requires the following well-known lemma (see [2]):

LEMma 4.1. Let $K$ be a subset of $A(X)$ such that the intersections $K \cap A_{n}(X)$ are finite for all $n \in \omega$. Then $K$ is closed in $A(X)$. A similar result remains valid for subsets of $F(X)$.

PROPOSITION 4.2. The following conditions are equivalent for a space $X$ :

(a) the group $F(X)$ is almost metrisable;

(b) the group $A(X)$ is almost metrisable;

(c) the space $X$ is discrete.

Proof: Since $A(X)$ is a quotient of $F(X)$, the implication (a) $\Rightarrow$ (b) is obvious. It is also clear that the groups $A(X)$ and $F(X)$ over a discrete space $X$ are discrete. Therefore, it suffices to verify that (b) implies (c).

Let $K$ be a non-empty compact set of countable character in $A(X)$. Since translates in topological groups are homeomorphisms, we can assume without loss of generality that $K$ contains the identity 0 of $A(X)$. Choose a countable base $\left\{U_{n}: n \in \omega\right\}$ for $A(X)$ at the set $K$. Define by induction a sequence $\left\{V_{n}: n \in \omega\right\}$ of open symmetric neighbourhoods of 0 in $A(X)$ such that $2 V_{n+1} \subseteq U_{n} \cap V_{n}$ for each $n \in \omega$. Then $L=\bigcap_{n \in \omega} V_{n}$ is a closed subgroup of $A(X)$ and $L \subseteq K$. Therefore, the subgroup $L$ of $A(X)$ is compact. Then one can apply Dudley's theorem in [4] to conclude that $L=\{0\}$, or argue as follows. If the group $L$ is non-trivial, take an element $g \in L \backslash\{0\}$ and consider the infinite cyclic subgroup $\langle g\rangle$ of $L$ generated by $g$. Then the intersection $A_{n}(X) \cap\langle g\rangle$ is finite for each $n \in \omega$, so the group $\langle g\rangle$ is discrete and closed in $L$ by Lemma 4.1. This contradiction shows that the group $L$ is trivial. Hence the identity 0 has countable pseudocharacter in $A(X)$ and in $K$. Since $K$ is compact, we conclude that $\chi(0, K) \leqslant \omega$. Therefore, $\chi(0, A(X)) \leqslant \chi(0, K) \cdot \chi(K, A(X)) \leqslant \omega$ by [5, 3.1.E]. In other words, the identity 0 has countable character in $A(X)$, so $X$ is discrete by Graev's theorem in [6]. 


\section{OPEN PROBLEMS}

Given a continuous mapping $f: X \rightarrow Y$, we denote by $\hat{f}$ the extension of $f$ to a continuous homomorphism of $F(X)$ to $F(Y)$. The next problem is related to Proposition 2.15. Its solution can provide a "uniform" proof of the equivalence of certain topological properties of $F_{2}(X)$ and $A_{2}(X)$.

Problem 5.1. Characterise the continuous onto mappings $f: X \rightarrow Y$ such that the restriction $f_{2}=\widehat{f} \uparrow_{F_{2}(X)}$ of $F_{2}(X)$ onto $F_{2}(Y)$ is perfect. We raise the analogous problem for $f_{3}=\left.\widehat{f}\right|_{F_{3}(X)}$.

A space $X$ is called a $q$-space provided that every point $x \in X$ has a sequence $\left\{U_{n}: n \in \omega\right\}$ of neighbourhoods with the property that if $x_{n} \in U_{n}$ for each $n \in \omega$, then the set $\left\{x_{n}: n \in \omega\right\}$ has a cluster point in $X$. It is easy to see that $X$ is a $q$-space if and only if it can be covered by countably compact sets, all having countable character in $X$. Clearly, every space of pointwise countable type is a $q$-space, but not vice versa (take any countably compact space which fails to be of pointwise countable type).

Problem 5.2. Characterise the spaces $X$ such that $F_{2}(X)$ is a $q$-space. What about $F_{n}(X)$ for all $n \in \omega$ ? The Abelian case?

\section{REEERENCES}

[1] A.V. Arhangel'skii, Topological spaces and mappings, Remarks on topological groups, (in Russian) (Moscow Univ. Publ., Moscow, 1969).

[2] A.V. Arhangel'skii, 'Relations among the invariants of topological groups and their subspaces', Russian Math. Surveys 35 (1980), 1-23. Russian original in: Uspekhy Mat. Nauk 35 (1980), 3-22.

[3] A.V. Arhangel'skii, 'Classes of topological groups', Russian Math. Surveys 36 (1981), 151-174. Russian original in: Uspekhy Mat. Nauk 36 (1981), 127-146.

[4] R.M. Dudley, 'Continuity of homomorphisms', Duke Math. J. 28 (1961), 587-594.

[5] R. Engelking, General topology (Heldermann Verlag, Berlin, 1989).

[6] M.I. Graev, 'Free topological groups', Amer. Math. Soc. Translation 8 (1962), 305-364. Russian original in: Izvestiya Akad. Nauk SSSR Ser. Mat. 12 (1948), 279-323.

[7] J.P. Hardy, S.A. Morris and H.B. Thompson, 'Application of the Stone-Cech compactification to free topological groups', Proc. Amer. Math. Soc. 55 (1976), 160-164.

[8] C. Joiner, 'Free topological groups and dimension', Trans. Amer. Math. Soc. 220 (1976), 401-418.

[9] J. Mack, S.A. Morris and E.T. Ordman, 'Free topological groups and the projective dimension of locally compact Abelian group', Proc. Amer. Math. Soc. 40 (1973), 303-308.

[10] S.A. Morris, 'Varieties of topological groups and left adjoint functors', J. Austral. Math. Soc. 16 (1973), 220-227.

[11] P. Nickolas, Free topological groups and free products of topological groups, (PhD thesis) (University of New South Wales, Australia, 1976).

[12] E.C. Nummela, 'Uniform free topological groups and Samuel compactifications', Topology Appl. 13 (1982), 77-83. 
[13] B.A. Pasynkov, 'Almost metrizable topological groups', (in Russian), Dokl. Akad. Nauk. SSSR 161 (1965), 281-284.

[14] V. G. Pestov, 'Some properties of free topological groups', Moscow Univ. Math. Bull. 37 (1982), 46-49.

[15] V.G. Pestov and K. Yamada, 'Free topological groups on metrizable spaces and inductive limits', Topology Appl. 98 (1999), 291-301.

[16] M.E. Rudin, Lectures on set-theoretic topology, Conf. Series in Math. 23 (Amer. Math. Soc., Providence, 1975).

[17] O.V. Sipacheva, 'Free topological groups of spaces and their subspaces', Topology Appl. 101 (2000), 181-212.

[18] M.G. Tkachenko, 'Completeness of free Abelian topological groups', Soviet Math. Dokl. 27 (1983), 341-345. Russian original in: Dokl. Akad. Nauk. SSSR 269 (1983), 299-303.

[19] M.G. Tkachenko, 'The topology of free groups over compacta', (in Russian), in Mappings and Functors, (P.S. Alexandroff, Editor) (Moskov. Gos. Univ., Moscow, 1984), pp. 122-137.

[20] V.V. Uspenskij, 'On subgroups of free topological groups', Soviet Math. Dokl. 32 (1985), 847-849. Russian original in: Dokl. Akad. Nauk SSSR 285 (1985), 1070-1072.

[21] K. Yamada, 'Free Abelian topological groups and $k$-spaces', Glas. Math. Ser. III 31 (1996), 83-91.

[22] K. Yamada, 'Metrizable subspaces of free topological groups on metrizable spaces', Topology Proc. 23 (1998), 379-409.

School of Mathematics and Applied Statistics University of Wollongong

New South Wales 2522

Australia

e-mail: peter_nickolas@uow.edu.au
Departamento de Matemáticas

Universidad Autónoma Metropolitana

Av. San Rafael Atlixco \# 186

Col. Vicentina, C.P. 09340

Iztapalapa

Mexico, D.F.

e-mail: mich@xanum.uam.mx 